\title{
The epistemic significance of numerals
}

\author{
Jan Heylen
}

\begin{abstract}
The central topic of this article is (the possibility of) de re knowledge about natural numbers and its relation with names for numbers. It is held by several prominent philosophers that (Peano) numerals are eligible for existential quantification in epistemic contexts ('canonical'), whereas other names for natural numbers are not. In other words, (Peano) numerals are intimately linked with de re knowledge about natural numbers, whereas the other names for natural numbers are not. In this article I am looking for an explanation of this phenomenon. It is argued that the standard induction scheme plays a key role.

Keywords Peano numerals - De re knowledge about numbers - Epistemic Arithmetic - ModalEpistemic Arithmetic - Arithmetical induction Explanation
\end{abstract}

\section{Introduction: de re knowledge about natural numbers and nu- merals}

Inspired by Russell (1905), Quine (1956) gives the following famous example involving the attitude of belief. Consider the following two sentences.

$$
\begin{aligned}
& (\exists x) \text { (Ralph believes that } x \text { is a spy). } \\
& \text { Ralph believes that }(\exists x)(x \text { is a spy). }
\end{aligned}
$$

The first sentence is an example of a de re belief, whereas the second sentence is an example of a de dicto belief. Noting that most of us are not acquainted with spies while it is common knowledge that there are spies, Quine claims that (1) is false, whereas (2) is true. Building on Quine's example, Kaplan (1968) asks us to suppose that there is one and only one shortest spy and that Ralph believes as much. Consider the following sentence.

$$
\text { Ralph believes that the shortest spy is a spy. }
$$

Based on the assumptions, (3) is quite plausible. In case the description 'the shortest spy' were eligible for existential quantification into belief contexts, one could derive (1) from (2) after all. Contrast this with the following sentence.

$$
\text { Ralph believes that J.B. Ortcutt is a spy. }
$$

According to Kaplan, one can infer (1) from (4) if 'J.B. Ortcutt' belongs to a class of names that bring Ralph into an intimate connection with the person Ortcutt. The details of Kaplan's proposal do not matter here. Kaplan's negative conclusion is that not all individual terms are eligible for existential quantification in belief contexts. The positive proposal by Kaplan is that there exist special kinds of individual terms that can be used for quantifying in.

Kaplan mentions that for beliefs about numbers there is a special class of names that are eligible for existential quantification. He is not entirely explicit about which names these are, but in the preceding 
section on existential quantification in modal contexts he talks about 'standard names' and his chief examples are numerals. Although the text is not entirely unambiguous, ${ }^{1}$ Ackerman (1978) interprets Kaplan as making the further claim that any purely arithmetical term is eligible for existential generalisation in belief contexts. She argues that the claim is false based on a counterexample that involves perfect numbers, i.e. positive integers that are equal to the sum of their proper positive divisors. Consider the following two sentences.

$(\exists x)$ (John believes that $x$ is a perfect number).

John believes that $(\exists x)(x$ is a perfect number $)$.

It may very well happen that (6) is true, perhaps because John has heard a mathematician among his friends saying that there are perfect numbers. But he left the conversation before his friend could give some examples and John never tried to come up with some examples himself. In these circumstances (5) is false. Ackerman then uses Kaplan's own trick against him (as she reads him). John has some minimal knowledge about natural numbers. In particular, he knows that, if there are perfect numbers, then there is a smallest perfect number. According to this story, the following is true.

John believes that the smallest perfect number is a perfect number.

If Ackerman's reading of Kaplan is correct, then he is committed to inferring (5) from (7). She rejects the soundness of this inference. On the other hand she is willing to accept that (5) can be inferred from:

John believes that six is a perfect number.

The negative conclusion by Ackerman is that not every arithmetical term (e.g. 'the least perfect number') is eligible for existential quantification in belief contexts. Her positive view is that the numerals, e.g. ' 6 ', are canonical in this respect.

Carnap (1956, p. 76-78) and Kripke (2011, p. 261, 344) have developed a line of thought that supports Ackerman's position on the eligibility of terms in doxastic contexts. Suppose you want to know which natural number has a certain property. The answer that is 'the natural number that is bigger than zero and identical to the product of that number by itself' can be met with the counterquestion 'Yes, but which number is that?'. It provides merely an indirect answer. It is only when one has proved that the description is co-denoting with 'one' that one has given the final, complete, direct answer. Similarly, the answer that it is identical to the value of the function that maps every number to the product of that number by itself and with one as its argument is merely an indirect answer. It is only when it has been calculated that the value of the function with that argument is 'one' that the final, complete, direct answer has been given. In Kripke's terminology a term is a buck stopper if no further calculation or proof is needed to determine its referent. Kripke (2011, p. 344) also notes that in computability theory a computation only stops when one reaches a numeral. The same point is made in (Boolos et al, 2003, p. 24). In complexity theory one also studies functions that are computable in the previous sense but with limitations on the length of computations.

There is disagreement among the cited philosophers as to which numeral systems are are canonical, i.e., the numerals belonging to that system are existentially generalisable in doxastic contexts. Ackerman (1978, p. 151) is very liberal. She explicitly acknowledges binary numerals, decimal, Hindu-Arabic

\footnotetext{
${ }^{1}$ In the main text of the section on the problem of quantifying into modal contexts Kaplan (1968, sec. VIII) talks exclusively about the numerals, but in a footnote Kaplan (1968, fn. 18) mentions Carnap (1956, p. 78)'s L-determinate designators, which are those designators $d$ that are L-equivalent to a Peano numeral $\bar{n}$, i.e. $d=\bar{n}$ belongs to every state-description. (The Peano numerals are composed of the symbol for zero and the symbol for the successor function.) The set of L-determinate designators includes all purely arithmetical terms (in the form of individual descriptions), e.g. 'the product of three and four'. After having proposed to allow only standard names in the set of terms that are exportable in modal contexts, Kaplan (1968, p. 197) suggests that 'the same trick' would work for doxastic contexts. Ackerman $(1978$, p. 147) thinks that that 'the same trick' refers to the restriction to standard names, but it may just as well have referred to a subset of the L-determinate designators, namely the Peano numerals.
} 
numerals and Roman numerals among the buck stoppers. Kripke thinks that the decimal numerals are epistemically privileged, whereas Ackerman is of the opinion that this position is open to the charge of ethnocentrism. Carnap (1956, p. 75) exclusively talks about Peano numerals as constituting the class of terms that can be used to provide direct, complete, final answers to questions about numbers. The Peano numerals are the symbol for zero $(\mathbf{0})$, the symbol for the successor function followed by the symbol for zero between brackets $(\mathbf{s}(\mathbf{0}))$, and so on. For reasons that will become clear, the focus in this article is on Peano numerals.

Not only is there the question about which numeral systems are canonical, there is also a problem noted by Kripke (Steiner, 2011, p. 165) and inspired by comments made by Wittgenstein (1978). In a nutshell the problem is that, whatever numeral system one adopts, small numerals may be buck stoppers but large numerals are not. For instance, the Peano numeral for 92648 is tens of pages long. By contrast, the Hindu-Arabic numeral for 92648 is just a handful of symbols. The former is not perspicuous to us, whereas the latter is. However, there are numbers for which the Hindu-Arabic numeral is also tens of pages long. The Wittgensteinian thought is that the result of a calculation has to be perspicuous. If that is correct, then large numerals are not buck stoppers. (Note that Wittgenstein's line of thinking goes against the notion of a buck stopper that is implicit in computability theory.) Kripke does not have a solution to this problem, although he does note that problem becomes acute more quickly with some notation systems than with others. It is an important problem and, alas, no solution is offered in this article. However, the conclusions reached in this article do constitute a constraint on possible solutions to the problem. In the remainder of the work I will proceed for the most part from the assumption that Peano numerals are indeed buck stoppers.

In Section 2 I will continue discussing the problem of de re knowledge about natural numbers, but this time within the framework of Peano Arithmetic. Then I set out to reach the two major goals of this article. First, I want to provide an explanation for the claim that Peano numerals are buck stoppers, i.e. direct, complete final answers to questions about numbers, or canonical terms, i.e. terms that are eligible for existential generalisation in doxastic contexts (Section 3). Second, I want to provide an explanation for the claim that other arithmetical terms (e.g., 'three times four') can be used to provide indirect answers to questions about numbers (Section 4). Although some of the results obtained apply to doxastic contexts in general, the main focus of this article is on epistemic contexts.

\section{De re knowledge about natural numbers and Peano numerals}

Let us first fix some terminology and notation, following (Boolos et al, 2003). I will use $\mathscr{L}_{\mathbf{A}}$ for the language of arithmetic. The set of Peano numerals is defined as follows: $\mathbf{0}$ is a Peano numeral; if $t$ is a Peano numeral, then so is $\mathbf{s}(t)$; nothing else is a Peano numeral. The set of terms of $\mathscr{L}_{\mathbf{A}}$ is defined as follows: if $x$ is a variable, it is a term of $\mathscr{L}_{\mathbf{A}} ; \mathbf{0}$ is a term of $\mathscr{L}_{\mathbf{A}}$; if $t$ and $t^{\prime}$ are terms of $\mathscr{L}_{\mathbf{A}}$, then so are $\mathbf{s}(t),\left(t+t^{\prime}\right),\left(t \times t^{\prime}\right)$; nothing else is a term of $\mathscr{L}_{\mathbf{A}}$. I will use $\mathscr{N}$ for the standard model of arithmetic, $\mathbb{N}$ for the set of natural numbers and $\bar{n}$ for the Peano numeral that denotes $n \in \mathbb{N}$ (in $\mathscr{N}$ ).

As noted in Section 1, the formal language $\mathscr{L}_{\mathbf{A}}$ with its Peano numerals is only one option. Another possibility consists in using the Hindu-Arabic numerals. However, there are two good reasons for choosing for $\mathscr{L}_{\mathbf{A}}$ and its Peano numerals. First, there are several axiomatic theories of arithmetic formulated in $\mathscr{L}_{\mathbf{A}}$. For instance, there is the axiomatic theory of minimal arithmetic, denoted $\mathbf{Q}$, the axiomatic theory of Robinson Arithmetic, denoted $\mathbf{R}$, and the axiomatic theory of Peano Arithmetic, denoted $\mathbf{P}$. Since it is one of the objectives of this article to provide deductive explanations of the epistemic significance of Peano numerals, it is convenient to have these axiomatic theories. Second, $\mathbf{P}$ contains a very important axiom scheme, namely the induction scheme:

IND $\phi(\mathbf{0} / x) \wedge \forall x(\phi \rightarrow \phi(\mathbf{s}(x) / x)) \rightarrow \forall x \phi$. 
It is clear from the outset that there is a link between IND and Peano numerals: the scheme contains the Peano numeral for zero and the symbol for the successor function. This will play an important role later. I will return to the issue of other numeral systems in Subsection 3.4.

In order to understand the problem of de re knowledge about natural numbers and the significance of Peano numerals, consider the extension of $\mathscr{L}_{\mathbf{A}}$ with a knowledge operator $K$. A philosophical question is whether every arithmetical term is eligible for existential generalisation in epistemic contexts. In other words, the question is whether the following principle is materially sound:

EGt $\phi \rightarrow \exists x \phi^{\prime}$

with $\phi^{\prime}$ identical to $\phi$ except that $t$ is replaced in zero or more places by $x$ (with the usual provisos). The following is an instance of EGt:

$$
K \overline{2} \times \overline{6}=\overline{3} \times \overline{4} \rightarrow \exists x K \overline{2} \times \overline{6}=x .
$$

As mentioned in Section 2, Kaplan (1968) endorsed the material soundness of EGt (on Ackerman's reading of him), whereas Carnap (1956), Ackerman (1978) and Kripke reject it. Abstracting away from the problem of multiple numeral systems and the problem of large numerals, they all endorse the claim that the following principle is materially sound:

EGn $\phi \rightarrow \exists x \phi^{\prime}$

with $\phi^{\prime}$ identical to $\phi$ except that the Peano numeral $\bar{n}$ is replaced in zero or more places by $x$ (with the usual provisos). The following is an instance of EGn:

$$
K \overline{2} \times \overline{6}=\overline{12} \rightarrow \exists x K \overline{2} \times \overline{6}=x .
$$

An obvious follow-up question is what could justify the claim that Peano numerals are canonical, i.e. that Peano numerals are eligible for existential quantification in epistemic contexts. Let us have a brief look at what Carnap, Ackerman and Kripke have to say on the matter.

Carnap (1956, p. 75) notes that Peano numerals have two nice properties. The first property is that there is a one-to-one correspondence between $\mathbb{N}$ and the set of Peano numerals, i.e. for every $n \in \mathbb{N}$ there is one and only one Peano numeral such that it denotes $n$ in $\mathscr{N}^{2}{ }^{2}$ The second property is that, for any two Peano numerals, one can determine the order relation between the numbers they denote. A similar remark was made by Kaplan (1968, p. 195). It is indeed provable that, if $\mathscr{N} \models \bar{n}=\bar{m}, \mathscr{N} \models \bar{n}<\bar{m}$ or $\mathscr{N} \models \bar{m}<\bar{n}$, then $\vdash_{\mathbf{Q}} \bar{n}=\bar{m}, \vdash_{\mathbf{Q}} \bar{n}<\bar{m}$ and $\vdash_{\mathbf{Q}} \bar{m}<\bar{n}$ respectively, and the same holds for $\mathbf{R}$ (Boolos et al, 2003, sect. 16.2 and 16.4). Let us investigate whether these two properties might explain why Peano numerals are buck stoppers.

The first property may look promising at first, because the same number, e.g. 3, can be denoted by many arithmetical terms, e.g. $\mathbf{s}(\mathbf{0})+\mathbf{s}(\mathbf{0})+\mathbf{s}(\mathbf{0})$ and $\mathbf{s}(\mathbf{0})+\mathbf{s}(\mathbf{s}(\mathbf{0}))$, so there is in general no oneto-one correspondence between arithmetical terms and numbers. However, Carnap's criterion does not discriminate between Peano numerals on the one hand and the set $\mathscr{T}$ of terms defined as follows: $\mathbf{0} \in \mathscr{T}$; if $t \in \mathscr{T}$, then $(t+\mathbf{s}(\mathbf{0})) \in \mathscr{T}$; nothing else is in $\mathscr{T}$. There is a one-to-one correspondence between the $\mathscr{T}$-terms and the natural numbers. Yet, it is not clear that $\mathscr{T}$-terms can be used as direct answers to the questions about which numbers have a certain unique property. This becomes clearer when one considers more complex alternatives, e.g. the set $\mathscr{T}^{\prime}$ of terms defined as follows: $(\mathbf{0} \times \mathbf{0}) \in \mathscr{T}^{\prime}$; if $t \in \mathscr{T}^{\prime}$, then $\left(\left(t \times \mathbf{0}^{\mathbf{0}}\right)+\mathbf{0}^{\mathbf{0}}\right) \in \mathscr{T}^{\prime}$; nothing else is in $\mathscr{T}^{\prime}$. It is implausible that $\left(\left((\mathbf{0} \times \mathbf{0}) \times \mathbf{0}^{\mathbf{0}}\right)+\mathbf{0}^{\mathbf{0}}\right)$ is a buck stopper. Therefore, the first property does not explain why Peano numerals are buck stoppers.

\footnotetext{
${ }^{2}$ Informally and roughly, one can prove that every natural number is denoted by a Peano numeral by invoking the second-order induction axiom and one can prove that every natural number is denoted by at most one Peano numeral by using the axiom that says that the successor function is a one-to-one function.
} 
The second property is definitely a very interesting property from an epistemic point of view. However, order statements with any closed terms belonging to $\mathscr{L}_{A}$ are decidable as well. It is equally well provable that, if $\mathscr{N} \models t=t^{\prime}, \mathscr{N} \models t<t^{\prime}$ or $\mathscr{N} \models t^{\prime}<t$, then $\vdash_{\mathbf{Q}} t=t^{\prime}, \vdash_{\mathbf{Q}} t<t^{\prime}$ and $\vdash_{\mathbf{Q}} t^{\prime}<t$ respectively, and the same holds for $\mathbf{R}$. To return to the examples given above, one can prove in $\mathbf{Q}$ and $\mathbf{R}$ that that $(\mathbf{0}+\mathbf{s}(\mathbf{0}))<(\mathbf{0}+\mathbf{s}(\mathbf{0})+\mathbf{s}(\mathbf{0}))$ and that $(\mathbf{0} \times \mathbf{0})<\left(\left((\mathbf{0} \times \mathbf{0}) \times \mathbf{0}^{\mathbf{0}}\right)+\mathbf{0}^{\mathbf{0}}\right)$.

In addition to the two mentioned properties, Carnap brings to the attention of the reader that the natural numbers form a progression, i.e. a discrete linear order with an initial element but no final element:

$$
0,1,2,3, \ldots
$$

Ackerman (1978, p. 151) remarks that understanding a numeral is sufficient for knowing which number it refers to. Understanding a numeral depends on understanding a system of numerals. The latter involves knowing how to generate the progression of numerals:

$$
\mathbf{0}, \mathbf{s}(\mathbf{0}), \mathbf{s}(\mathbf{s}(\mathbf{0})), \mathbf{s}(\mathbf{s}(\mathbf{s}(\mathbf{0}))), \ldots
$$

The know-how needed to generate the above sequence consists in knowing the recursive definition of Peano numerals and how to repeatedly apply the recursive step. Likewise, knowing the recursive definitions of terms belonging to $\mathscr{T}$ and $\mathscr{T}^{\prime}$ and knowing how to repeatedly apply the respective recursive steps enables one to produce the respective sequences:

$$
\begin{gathered}
\mathbf{0}, \mathbf{0}+\mathbf{s}(\mathbf{0}), \mathbf{0}+\mathbf{s}(\mathbf{0})+\mathbf{s}(\mathbf{0}), \mathbf{0}+\mathbf{s}(\mathbf{0})+\mathbf{s}(\mathbf{0})+\mathbf{s}(\mathbf{0}), \ldots \\
\mathbf{0} \times \mathbf{0},\left((\mathbf{0} \times \mathbf{0}) \times \mathbf{0}^{\mathbf{0}}\right)+\mathbf{0}^{\mathbf{0}},\left(\left(\left((\mathbf{0} \times \mathbf{0}) \times \mathbf{0}^{\mathbf{0}}\right)+\mathbf{0}^{\mathbf{0}}\right) \times \mathbf{0}^{\mathbf{0}}\right)+\mathbf{0}^{\mathbf{0}}, \ldots
\end{gathered}
$$

Ackerman's remark comes down to noting that the successor relation on the domain of Peano numerals is decidable. But so is the successor relation on the domain of $\mathscr{T}$, and likewise for $\mathscr{T}^{\prime}$. Kripke seems to add complexity considerations to the debate: the successor relation should be 'transparent' (Steiner, 2011, p. 166), which can perhaps be understood as being easily decidable. But even this strengthening of Ackerman's criterion seems powerless to rule out the terms of $\mathscr{T}$ and $\mathscr{T}^{\prime}$.

Saul Kripke has an interesting take on the problem. His work on the subject remains unpublished to this date, but Steiner (2011) gives an exposition of his views. The core of Kripke's position is summarized by Steiner (2011, p. 168) as follows:

All notations, however, seem to be sequences in a finite alphabet, and these sequences we call "numerals". This suggests that the numbers themselves should be sequences, in order that the notation should be structurally descriptive of the objects denoted. The rules for manipulating the numerals (that is, the sequences) induce the abstract operations on the numbers. The algorithm for finding the next numeral imposes the definition of "successor" on the "denotations" of these numerals. Just as we fix the notion of an algorithm and then see what functions are defined, so do we fix the notion of calculation as symbolic manipulation and then see what numbers there are. Small wonder that philosophers and lay people are confused concerning the difference between numerals and numbers.

This is what I mean, then, by "quasi-nominalism." The identity of the numbers is induced by the properties of the notation, and thus their existence is, in some sense, derivative.

Let us apply this to the system of Peano numerals. In the beginning there were Peano numerals and they were ordered as a progression - see (12). Then came the Peano numbers, which are also ordered as a progression:

$$
0, s(0), s(s(0)), s(s(s(0))), \ldots
$$

The symbol 0 denotes the number zero and the symbol $s$ denotes the successor function on $\mathbb{N}$. Since Peano numbers depend on Peano numerals, the latter are canonical names for the former. This idea is related to the claim that the domains of the intended models of arithmetic are constituted by naming systems 
with recursive operations on them that satisfy the Peano axioms (Halbach and Horsten, 2005). There is ongoing discussion about the proposal (Quinon and Zdanowski, 2007; Button and Smith, 2011; Horsten, 2012; Dean, 2013; Quinon, 2014). One major goal of this article is to shed some light on the question why Peano numerals are canonical. Nothing about the nature of numbers will be assumed. Therefore, the investigation carried out here is complementary to pursuing the line of inquiry of Kripke and Halbach and Horsten (2005). Section 3 is devoted to this topic.

Although $\overline{3} \times \overline{4}$ may not be a canonical term or buck stopper, it does provide an indirect answer to the question about the identity of the number denoted by $\overline{2} \times \overline{6}$. The notion of a direct answer was cashed out in terms of eligibility for existential generalisation in epistemic contexts. One can do something similar with the notion of an indirect answer. For this purpose consider the extension of $\mathscr{L}_{\mathbf{A}} \cup\{K\}$ with a possibility operator $\diamond$. In this language one can express the notion of knowability. The following definition singles out a fragment of $\mathscr{L}_{\mathbf{A}} \cup\{\diamond, K\}$, namely the set of formulas in which the possibility operator (if it occurs at all) is always followed by the knowledge operator and in which the knowledge operator (if it occurs at all) is always preceded by the possibility operator.

Definition 2.1. Let $\tau$ be a fragment of $\mathscr{L}_{\mathbf{A}} \cup\{\diamond, K\}$ defined as follows:

1. if $\phi$ is an atomic formula, then $\phi \in \tau$;

2. if $\phi=\neg \psi$ and $\psi \in \tau$, then $\phi \in \tau$;

3. if $\phi=\psi \rightarrow \theta$ and $\psi, \theta \in \tau$, then $\phi \in \tau$;

4. if $\phi=\forall x \psi$ and $\psi \in \tau$, then $\phi \in \tau$;

5. if $\phi=\diamond K \psi$ and $\psi \in \tau$, then $\phi \in \tau$;

6. nothing else is an element of $\tau$.

Now consider whether for a term $t \in \mathscr{L}_{\mathbf{A}}$ and for any $\phi \in \tau$, it is the case that $\phi \rightarrow \exists x \phi^{\prime}$, with $\phi^{\prime}$ identical to $\phi$ except that $t$ is replaced in zero or more places by $x$ (assuming that $t$ is substitutable for $x$ in $\phi$ ). If yes, then $t$ is at the least an indirect answer. (If $t$ is also eligible for existential generalisation in any context $\phi \in \mathscr{L}_{A} \cup\{K\}$, then it is a direct answer as well.) If no, then $t$ is not even an indirect answer. The claim that $\overline{3} \times \overline{4}$ is an indirect answer to the question about the identity of the number denoted by $\overline{2} \times \overline{6}$ is then expressed by claiming that the following is true:

$$
\diamond K \overline{2} \times \overline{6}=\overline{3} \times \overline{4} \rightarrow \exists x \diamond K \overline{2} \times \overline{6}=x .
$$

If one has the $\mathbf{T}$ system of modal logic in the background, then $\diamond K \overline{2} \times \overline{6}=\overline{3} \times \overline{4}$ is implied by $K \overline{2} \times \overline{6}=$ $\overline{3} \times \overline{4}$. So, knowing that $\overline{2} \times \overline{6}$ is identical to $\overline{3} \times \overline{4}$ entails possible de re knowledge about the number denoted by $\overline{2} \times \overline{6}$. The second major goal of this article is to illuminate the question why arithmetical terms other than Peano numerals can be used to provide indirect answers to questions about which number has a certain unique property. This is the subject of Section 4.

\section{Peano numerals, canonicity and induction}

Let us refer to terms that are eligible for existential generalisation in epistemic contexts as canonical terms. Subsection 3.1 is dedicated to the proof that Peano numerals are canonical terms, if the knowledge operator is allowed in the induction scheme. Subsection 3.2 is dedicated to the proof that other arithmetical terms are not canonical, even if one allows the knowledge operator in the induction scheme and one adds certain epistemic, modal and modal-epistemic principles. Subsection 3.3 zooms in on the relation between the (non-)canonicity of arithmetical terms and the induction scheme. Subsection 3.4 contains a summary and provides some final reflections on the kind of explanation of the canonicity of Peano terms that is offered in this section. 


\subsection{Peano terms are canonical}

The theory used in this subsection is $\mathbf{P}$ formulated in $\mathscr{L}_{\mathbf{A}} \cup\{K\}$ and is denoted as $\mathbf{P}^{K-}$. The minus sign indicates that it does not contain the unrestricted universal instantiation scheme:

UIt $\forall x \phi \rightarrow \phi(t / x)$ (where $t$ is substitutable for $x$ in $\phi$ ).

Indeed, UIt is logically equivalent to EGt and, as was discussed in Section 1 and 2, EGt is not philosophically sound. So, we have reasons to reject UIt as well. Instead of UIt the axiomatic base of the logical fragment of $\mathbf{P}^{K-}$ contains two restricted universal instantiation schemes, viz.:

UI $\forall x \phi \rightarrow \phi(y / x) ;$

RUIt $\forall x \phi \rightarrow \phi(t / x)$ for all $t, \phi \in \mathscr{L}_{\mathbf{A}}$.

Scheme UI is the version of UIt in which the range of $t$ is restricted to variables and scheme RUIt is the version of UIt in which the range of $\phi$ and $t$ are restricted to formulas and terms of $\mathscr{L}_{\mathbf{A}}$. The reason for adding UI to the axiomatic base is that there is an intimate conceptual link between quantifiers and variables. The reason for adding RUIt is that it is needed for the full deductive power of $\mathbf{P}$, restricted to $\mathscr{L}_{\mathbf{A}}$, e.g. one need to be able to deduce $\mathbf{s}(\mathbf{0})+\mathbf{s}(\mathbf{0})=\mathbf{s}(\mathbf{0}+\mathbf{s}(\mathbf{0}))$ from $\forall x \forall y(\mathbf{s}(x)+y=\mathbf{s}(x+y))$.

It is presupposed that the axiom of self-identity, the axiom scheme of the substitutivity of identicals and the rule of universal generalization are formulated with individual variables as well:

SI $x=x$;

SUB $x=y \rightarrow\left(\phi \rightarrow \phi^{\prime}\right)$, with $\phi^{\prime}$ identical to $\phi$, except that zero or more occurrences of free $x$ are replaced with free $y$.

UG If $\vdash \phi \rightarrow \psi$ and $x$ does not occur freely in $\phi$, then $\vdash \phi \rightarrow \forall x \psi$.

If one had UIt, then it would follow that

SUBt $t=t^{\prime} \rightarrow\left(\phi \rightarrow \phi^{\prime}\right)$, with $\phi^{\prime}$ identical to $\phi$, except that zero or more occurrences of $t$ are replaced with $t^{\prime}$ (assuming $t^{\prime}$ is substitutable for $t$ in $\phi$ ).

But it is not generally correct that, if $\overline{2} \times \overline{3}=\overline{3} \times \overline{4}$ and if one knows that $\overline{2} \times \overline{3}=\overline{2} \times \overline{3}$, then one knows that $\overline{2} \times \overline{3}=\overline{3} \times \overline{4}$. Conversely, one can deduce UIt from UI and SUBt. Therefore, the substitution principle belonging to $\mathbf{P}^{K-}$ is formulated with variables rather than terms. Note that the same relation holds between RUIt and the following principle:

RSUBt $t=t^{\prime} \rightarrow\left(\phi \rightarrow \phi^{\prime}\right)$, for all $\phi \in \mathscr{L}_{\mathbf{A}}$ and with $\phi^{\prime}$ identical to $\phi$, except that zero or more occurrences of $t$ are replaced with $t^{\prime}$ (assuming $t^{\prime}$ is substitutable for $t$ in $\phi$ ).

In other words, arithmetic is extensional. Moving to the arithmetical fragment of $\mathbf{P}^{K-}$, it is crucial to note that the $K$-operator is allowed in instances of the induction scheme. Also noteworthy is that no specific principle about $K$ is postulated that restricts its interpretation and, in particular, no principle is put forward that excludes reading $K$ as a doxastic operator.

The main question of this subsection is whether Peano numerals are eligible for universal instantiation (and existential generalization) in epistemic contexts. To be more specific, the question is whether the following scheme can be proved in $\mathbf{P}^{K-}$.

UIn $\forall x \phi \rightarrow \phi(\bar{n} / x)$ 
Indeed, EGn is logically equivalent to UIn. The main result of this subsection is an affirmative answer to that question. In fact, something stronger is proved. Peano numerals are closed terms, but Peano terms are open or closed. Define the set of open or closed Peano terms inductively as follows: 0 is a Peano term; if $t$ is a variable, then $t$ is a Peano term; if $t$ is a Peano term, then so is $\mathbf{s}(t)$; nothing else is a Peano term. It will be proved that $\forall x \phi \rightarrow \phi(t / x)$ for any Peano term $t$ (provided that $t$ is substitutable for $x$ in $\phi)$. First one needs to prove two lemmas.

Lemma 3.1. $\vdash_{\mathbf{p}^{K-}} \forall x(x=\mathbf{0} \rightarrow(\phi \leftrightarrow \phi(\mathbf{0} / x)))$.

Proof. The proof is by the induction principle.

First, one needs to prove that

$$
\mathbf{0}=\mathbf{0} \rightarrow\left(\phi^{\prime} \leftrightarrow \phi^{\prime}(\mathbf{0} / x)\right),
$$

with $\phi^{\prime}=\phi(\mathbf{0} / x)$. But this is trivial, since $\phi^{\prime}(\mathbf{0} / x)$ is identical to $\phi^{\prime}$.

Second, one needs to prove that

$$
\forall x\left((x=\mathbf{0} \rightarrow(\phi \leftrightarrow \phi(\mathbf{0} / x))) \rightarrow\left(\mathbf{s}(x)=\mathbf{0} \rightarrow\left(\phi(\mathbf{s}(x) / x) \leftrightarrow \phi^{\prime}(\mathbf{s}(x) / x)\right)\right)\right),
$$

with $\phi^{\prime}$ identical to $\phi(\mathbf{0} / x)$. But this is trivial again, since it is an axiom of $\mathbf{P}$ that $\forall x \mathbf{s}(x) \neq \mathbf{0}$.

Lemma 3.2. $\vdash_{\mathbf{P}^{K-}} \forall x \forall y(x=\mathbf{s}(y) \rightarrow(\phi \leftrightarrow \phi(\mathbf{s}(y) / x)))$ (assuming that $\mathbf{s}(y)$ is substitutable for $x$ in $\left.\phi\right)$.

Proof. The proof is by the induction principle.

First, one needs to prove that

$$
\forall y\left(\mathbf{0}=\mathbf{s}(y) \rightarrow\left(\phi^{\prime} \leftrightarrow \phi^{\prime}(\mathbf{s}(y) / \mathbf{0})\right)\right),
$$

with $\phi^{\prime}=\phi(\mathbf{0} / x)$. This is trivial, since it is a theorem of $\mathbf{P}$ that $\mathbf{0} \neq \mathbf{s}(y)$.

Second, one needs to prove that for all $x$, if

$$
\forall y(x=\mathbf{s}(y) \rightarrow(\phi \leftrightarrow \phi(\mathbf{s}(y) / x))),
$$

then

$$
\forall y\left(\mathbf{s}(x)=\mathbf{s}(y) \rightarrow\left(\phi(\mathbf{s}(x) / x) \leftrightarrow \phi^{\prime}(\mathbf{s}(x) / x)\right)\right),
$$

with $\phi^{\prime}$ identical to $\phi(\mathbf{s}(y) / x)$. Suppose that $\mathbf{s}(x)=\mathbf{s}(y)$. It follows by an axiom scheme of $\mathbf{P}$ that $x=y$. Suppose that $\phi(\mathbf{s}(x) / x)$. It follows by SUB that $\phi(\mathbf{s}(y) / x)$, which is identical to $\phi^{\prime}(\mathbf{s}(x) / x)$, since $\phi^{\prime}$ does not contain any free occurrences of $x$ left to be substituted. And vice versa.

Theorem 3.3. $\vdash_{\mathbf{P}^{K}-} \forall x \phi \rightarrow \phi(t / x)$ for every Peano term $t$ (where $t$ is substitutable for $x$ in $\phi$ ).

Proof. The proof is by induction on the complexity of Peano terms $t$. The case of $t=x$ is trivial. I will only prove the two non-trivial cases.

First case: $t=\mathbf{0}$. Suppose that $\forall x \phi$. It is a theorem of $\mathbf{P}$ that $\exists y(y=\mathbf{0})$, for some $y$ not in $\forall x \phi$. Suppose that $y=\mathbf{0}$. Axiom scheme UI says that $\forall x \phi \rightarrow \phi(y / x)$. Then $\phi(y / x)$. On the basis of Lemma 3.1 and UI one can deduce that $\phi(\mathbf{0} / x)$.

Second case: $t=\mathbf{s}\left(t^{\prime}\right)$, for some Peano term $t^{\prime}$. Suppose that $\forall x \phi$. It is a theorem of $\mathbf{P}$ that

$$
\exists y\left(y=\mathbf{s}\left(t^{\prime}\right)\right),
$$

for some $y$ not in $\forall x \phi$ and not in $\mathbf{s}\left(t^{\prime}\right)$. Suppose that $y=\mathbf{s}\left(t^{\prime}\right)$. Axiom scheme UI says that $\forall x \phi \rightarrow \phi(y / x)$. Then $\phi(y / x)$. The induction hypothesis is that $\forall x \phi \rightarrow \phi\left(t^{\prime} / x\right)$, for all $\phi$. Therefore, one can use $t^{\prime}$ to universally instantiate Lemma 3.2, yielding:

$$
y=\mathbf{s}\left(t^{\prime}\right) \rightarrow\left(\phi(y / x) \leftrightarrow \phi\left(\mathbf{s}\left(t^{\prime}\right) / x\right)\right) .
$$

It follows that $\phi\left(\mathbf{s}\left(t^{\prime}\right) / x\right)$. 
The philosophical significance of Theorem 3.3 consists in the fact that allowing a knowledge operator in the induction scheme suffices to prove that Peano terms are eligible for universal instantiation in epistemic contexts, i.e. Peano terms are canonical terms, even if the axiom scheme for universal instantiation is severely restricted. There are two important elements here. First, one does not need to assume the canonicity of Peano terms, but one can prove it. Second, it is the induction scheme that does the work. The induction scheme contains the Peano numeral for the number zero and the symbol for the successor function. This ties in nicely with the philosophical insight that Peano numerals are epistemically privileged.

In Subsection 3.3 the role of the induction principle will be more closely investigated. Before taking up that task, one needs to check whether or not arithmetical terms other than the Peano terms are canonical. This is the topic of the next subsection.

\subsection{Arithmetical terms other than the Peano terms are not canonical}

In Subsection 3.1 it was proved that Peano terms are canonical according to $\mathbf{P}^{K-}$. A natural follow-up question is whether or not other arithmetical terms are canonical according to $\mathbf{P}^{K-}$. The desired answer is negative, as explained in Section 2. But a negative answer to the question about $\mathbf{P}^{K-}$ does not suffice. After all, it may be that a deductively stronger theory formulated in an extended language does prove that arithmetical terms other than the Peano terms are canonical. Let us consider one such framework.

For present purposes I will make use of a weak version of Modal-Epistemic Arithmetic (Horsten, 1994, 1998), which I call MEP $\mathbf{P}^{\diamond K-3}$ The latter is the extension of $\mathbf{P}$, formulated in $\mathscr{L}_{\mathbf{A}} \cup\{\diamond, K\}$ rather than $\mathscr{L}_{\mathbf{A}}$, with modal system $\mathbf{S 5}$ and one epistemic and one modal-epistemic principle:

FACT $K \phi \rightarrow \phi$.

COMP $\Gamma \vdash \phi \Rightarrow(K) \Gamma \vdash \diamond K \phi$, with $(K) \Gamma=\{K \psi \mid \psi \in \Gamma\}$.

The axiom scheme FACT expresses the factivity of knowledge. The axiom scheme COMP expresses a form of logical competence: if an epistemic agent knows the premisses of a deductively valid argument, then that agent is able to know the conclusion of the argument. Logical omniscience is avoided, but so is logical ignorance.

If one cannot prove that arithmetical terms other than Peano terms are canonical according to $\mathbf{M E P} \mathbf{P}^{\diamond K-}$, then one has a reasonable case that one does not need to accept that those terms are canonical. The main result of this subsection is that this is indeed the case. As an example of a non-canonical term I will use $\mathbf{s}(\mathbf{0})+\mathbf{s}(\mathbf{0})$, which belongs to $\mathscr{T}$ (see Section 2).

Theorem 3.4. $\nvdash_{\mathbf{M E P}} \diamond K-\forall x K x=x \rightarrow K \mathbf{s}(\mathbf{0})+\mathbf{s}(\mathbf{0})=\mathbf{s}(\mathbf{0})+\mathbf{s}(\mathbf{0})$.

Proof. Let us first define an awareness interpretation of $\mathscr{L}_{\mathbf{A}} \cup\{\diamond, K\}$ of the type described in (Heylen, 2013). The interpretation $\mathscr{M}$ consists of the following elements:

1. $W=\left\{w_{1}, w_{2}\right\}$

2. $D=\mathbb{N}$;

3. $R_{M}=W^{2}$;

4. $R_{E}=\left\{\left\langle w_{1}, w_{1}\right\rangle,\left\langle w_{2}, w_{2}\right\rangle\right\}$;

\footnotetext{
${ }^{3}$ This theory is weaker than MEA, which was introduced in (Horsten, 1994). Two important differences are, first, that MEA contains UIt, whereas MEP $\mathbf{P}^{\diamond K}$ - contains only the restricted versions UI and RUIt, and second, that MEA contains a principle expressing closure of knowability under deducibility, whereas $\mathbf{M E P}^{\diamond K-}$ contains a weaker logical competence principle. The significance of the first difference is clear from the context here. The importance of the second difference is defended in (Heylen, 2014).
} 
5. $V$ is $\mathscr{N}$;

6. $S$ the set of variable assignment functions;

7. $A$ is a function from $W$ and $S$ to $\wp(\mathscr{L} \cup\{\diamond, K\})$, defined as follows:

(a) for any variable assignment $a, A\left(w_{1}, a\right)=\mathscr{L}_{\mathbf{A}} \cup\{\diamond, K\}$;

(b) for any variable assignment $a, A\left(w_{2}, a\right)=\left\{t=t^{\prime} \mid\right.$ den $\left._{\mathscr{N}, a}(t)=\operatorname{den}_{\mathscr{N}, a}\left(t^{\prime}\right)\right\}$, with $t, t^{\prime}$ Peano terms and with $\operatorname{den}_{\mathscr{N}, a}(t)$ the denotation of $t$ relative to the standard interpretation of arithmetic, $\mathscr{N}$, and an assignment $a$.

The denotation of a term relative to $\mathscr{M}, w$ and $a$ (with $w \in W$ and $a \in A$ ) is straightforward: all arithmetical symbols are given their standard interpretation and the denotation of terms relative to $\mathscr{M}$ and $a$ (with $a \in A)$ is as usual.

Satisfaction of a well-formed formula $\phi$ relative to $\mathscr{M}, w \in W$ and $a \in A$ is defined as follows:

1. if $\phi$ is an atomic formula, then the clause is as usual;

2. if $\phi$ is of the forms $\neg \psi, \psi \wedge \theta, \forall x \psi$, then the clauses are as usual;

3. if $\phi$ is of the form $\diamond \psi$, then $\mathscr{M}, w, a \models \phi$ if and only if $\mathscr{M}, w^{\prime}, a \models \psi$ for every $w^{\prime}$ such that $w R_{M} w^{\prime}$;

4. if $\phi$ is of the form $K \psi$, then $\mathscr{M}, w, a=\phi$ if and only if $\psi \in A(w, a)$ and $\mathscr{M}, w^{\prime}, a \models \psi$ for every $w^{\prime}$ such that $w R_{E} w^{\prime}$.

One needs to check whether $\mathscr{M} \models \mathbf{M E P} \mathbf{P}^{\diamond K-}$. The proof is by induction on the complexity of proof in $\mathbf{M E P}^{\diamond K-}$. I will only sketch the proof. It is the case that $\mathscr{M} \models \mathbf{S 5}$, since $R_{M}$ is an equivalence relation. It is also the case that $\mathscr{M} \models F A C T$, since $R_{E}$ is a reflexive relation. In addition, $\mathscr{M} \models C O M P$, since $A\left(w_{1}, a\right)=\mathscr{L}_{\mathbf{A}} \cup\{\diamond, K\}$ for all variable assignments $a$ and since $w R_{M} w_{1}$ for all $w \in W$.

To prove that UI, RUIt, UG, SI and SUB are sound on the model, I will make use of a few lemmas. First, one can easily prove by induction on the complexity of formulas the following principle of replacement.

PR1 For all $\phi \in \mathscr{L}_{\mathbf{A}}$, for all worlds $w$ and assignments $a$, for any variable $x$ and term $t$, it is the case that $M, w, a \models \phi(t / x)$ if and only if $M, w, a\left[x \mapsto \operatorname{den}_{\mathscr{M}, a}(t)\right] \models \phi$ (assuming that $t$ is substitutable for $x$ in $\phi$ ), where $a\left[x \mapsto \operatorname{den}_{\mathscr{M}, a}(t)\right]$ is identical to $a$, except perhaps that $a\left[x \mapsto \operatorname{den}_{\mathscr{M}, a}(t)\right]$ maps $x$ to the denotation of $t$ relative to $a$.

Consequently, RUIt is sound on the model. We also need another version of the principle of replacement to prove that UI is sound on the model.

PR2 For all $\phi \in \mathscr{L}_{\mathbf{A}} \cup\{\diamond, K\}$, for all worlds $w$ and assignments $a$, for any variables $x, y$, it is the case that $M, w, a \models \phi(y / x)$ if and only if $M, w, a[x \mapsto a(y)] \models \phi$, where $a[x \mapsto a(y)]$ is identical to $a$, except perhaps that $a[x \mapsto a(y)]$ maps $x$ to $a(y)$. The proof is also by induction on the complexity of formulas.

The only interesting case is when $\phi=K \psi$. The subcase of $w_{1}$ is proved by noting that $A\left(w_{1}, a\right)=$ $A\left(w_{1}, a[x \mapsto a(y)]\right)$. The subcase of $w_{2}$ is easy as well. Note that $\mathscr{M}, w_{2}, a=t=t^{\prime}(y / x)$ if and only if $\mathscr{M}, w_{2}, a[x \mapsto a(y)] \models t=t^{\prime}$. The proof is by induction on the complexity of terms and the definition of $a[x \mapsto a(y)]$. The definition of $A\left(w_{2}, a\right)$ and the resulting definition of $A\left(w_{2}, a[x \mapsto a(y)]\right)$ do the rest. In order to prove UG one has to prove a principle of agreement.

PA For all $\phi \in \mathscr{L}_{\mathbf{A}} \cup\{\oslash, K\}$, for all worlds $w$ and assignments $a$ and $a^{\prime}$, for any variables $x, y$, it is the case that, if $a$ and $a^{\prime}$ agree on the free variables in $\phi$, then $M, w, a \models \phi$ if and only if $M, w, a^{\prime} \models \phi$. 
The only interesting case is when $\phi=K \psi$. The subcase of $w_{1}$ is proved by noting that $A\left(w_{1}, a\right)=$ $A\left(w_{1}, a^{\prime}\right)$. The subcase of $w_{2}$ is proved by noting that, for all terms $t$, if $t$ occurs in $\psi$, then den $_{\mathscr{M}}, a(t)=$ den $_{\mathscr{M}, a^{\prime}}(t)$. Hence, for all Peano terms $t$ and $t^{\prime}$ occurring in $\psi, t=t^{\prime} \in A\left(w_{2}, a\right)$ if and only if $t=t^{\prime} \in$ $A\left(w_{2}, a^{\prime}\right)$. The soundness of SI is trivial. The soundness of SUB is proved by induction on the complexity of formulas. The only interesting case is when $\phi=K \psi$. The subcase of $w_{1}$ is trivial, given the definition of $A\left(w_{1}, a\right)$. The subcase of $w_{2}$ follows from the fact that, if $a(x)=a(y)$, then $\mathscr{M}, w, a \models t=t^{\prime}$ if and only if $\mathscr{M}, w, a \models t=t^{\prime}(y / x)$, with $t^{\prime}$ identical to $t$ except that zero or more occurrences of free $x$ have been replaced by free $y$. Consequently, for any terms $t_{1}, t_{2}$, it is the case that $t_{1}=t_{2} \in A\left(w_{2}, a\right)$ if and only if $t_{1}^{\prime}=t_{2}^{\prime} \in A\left(w_{2}, a\right)$.

The axioms of $\mathbf{P}$ are sound on the model, since the domain is $\mathbb{N}$ and the interpretation of the arithmetical vocabulary is standard. Finally, suppose that $\mathscr{M}, w, a \models \phi(\mathbf{0} / x)$ and that $\mathscr{M}, w, a \models \forall x(\phi \rightarrow \phi(\mathbf{s}(x) / x))$ and assume for a reductio ad absurdum that $\mathscr{M}, w, a^{\prime} \models \neg \phi(x)$ for some $x$-variant $a^{\prime}$ of $a$. The proof makes use of yet a third replacement principle.

PR3 For all $\phi \in \mathscr{L}_{\mathbf{A}} \cup\{\diamond, K\}$, for all worlds $w$ and assignments $a$, for any variables $x$ and for any Peano term $t$, it is the case that $M, w, a \models \phi(t / x)$ if and only if $M, w, a\left[x \mapsto \operatorname{den}_{\mathscr{M}, a}(t)\right] \models \phi$.

The proof is essentially the same as the proof of the second replacement principle. Next, suppose that there is a $n \in \mathbb{N}$ such that $a^{\prime}(x)=n$ and $\mathscr{M}, w, a^{\prime} \models \neg \phi$. In case $n=0$ one can use the replacement principle to derive that $\mathscr{M}, w, a=\phi(\mathbf{0} / x)$, since $a$ and $a^{\prime}$ are $x$-variants and $x$ is not free in $\phi(\mathbf{0} / x)$. Subsequently, one can use the third replacement principle to derive $\mathscr{M}, w, a^{\prime} \models \phi$. Contradiction. In case $n \neq 0$ there is an $m \in \mathbb{N}$ such that $n=s(m)$. It is convenient to denote $a^{\prime}$ as $a_{n}$. Now consider $a_{n-1}$, i.e. the $x$-variant of $a$ that assigns $m$ to $x$. Since $a_{n-1}$ is an $x$-variant of $a$, it follows that $\mathscr{M}, w, a_{n-1} \models \phi \rightarrow \phi(\mathbf{s}(x))$. It is a consequence of the third replacement principle that $\mathscr{M}, w, a_{n-1} \models \phi(\mathbf{s}(x))$ if and only if $\mathscr{M}, w, a_{n-1}[x \mapsto$ $\left.\operatorname{den}_{M, a_{n-1}}(\mathbf{s}(x))\right] \models \phi$. But $a_{n-1}\left[x \mapsto \operatorname{den}_{M, a_{n-1}}(\mathbf{s}(x))\right]$ is just $a_{n}$. Therefore, $\mathscr{M}, w, a_{n-1} \models \neg \phi$. Repeat this reasoning until it has been derived that $\mathscr{M}, w, a_{n-n}=\neg \phi$. Then reason as in the case of $n=0$. Contradiction.

It is easy to check that $\mathscr{M}, w_{2}, a \models \forall x K x=x$, but $\mathscr{M}, w_{2}, a \not \models K \mathbf{s}(\mathbf{0})+\mathbf{s}(\mathbf{0})=\mathbf{s}(\mathbf{0})+\mathbf{s}(\mathbf{0})$.

If one is allowed to instantiate the induction scheme with formulas containing the knowledge operator, then one can prove that Peano terms are canonical. Even if one adds certain modal, epistemic and modalepistemic principles one cannot prove that other arithmetical terms are canonical. In the next subsection the relation between the induction scheme and these (non-)canonicity results will be subjected to closer scrutiny.

\subsection{Induction and canonicity}

In Subsection 3.1 it was shown that allowing the knowledge operator in the induction scheme was sufficient for proving that Peano terms are canonical. In Subsection 3.2 it was shown that other arithmetical terms are not similarly provably canonical. One possible line of explanation is that what is at the root of this phenomenon is that the classical induction scheme contains the symbol for zero and that it contains the symbol for the successor function. In Subsubsection 3.3.1 further evidence for this possible explanation is considered, whereas in Subsubsection 3.3.2 counterevidence is presented. Additionally, the non-extensionality of the theories that are investigated is highlighted.

\subsubsection{Induction scheme without the successor symbol and canonicity}

The scheme IND is the classical induction scheme. Its antecedent and its consequent are respectively:

$$
\begin{array}{r}
\phi(\mathbf{0} / x) \\
\forall x(\phi \rightarrow \phi(\mathbf{s}(x) / x))
\end{array}
$$


If one had UIt or SUBt, then (16) would be provably equivalent to:

$$
\forall x(\phi \rightarrow \phi(x+\mathbf{s}(\mathbf{0}) / x)),
$$

given that $\vdash_{\mathbf{P}} \mathbf{S}(x)=x+\mathbf{s}(\mathbf{0})$. So, in extensional logic (in which SUBt is valid), IND is provably equivalent to:

$$
(\phi(\mathbf{0} / x) \wedge \forall x(\phi \rightarrow \phi(x+\mathbf{s}(\mathbf{0}) / x))) \rightarrow \forall x \phi .
$$

Let $\mathbf{P}^{K-\dagger}$ be identical to $\mathbf{P}^{K-}$, except that the classical induction scheme IND is replaced by (18). Furthermore, consider the set of terms $\mathscr{S}^{\dagger}$ defined inductively as follows: $\mathbf{0}$ belongs to $\mathscr{S}^{\dagger}$; if $t$ is a variable, then $t \in \mathscr{S}^{\dagger}$; if $t$ belongs to $\mathscr{S}^{\dagger}$, then so does $t+\mathbf{s}(\mathbf{0})$; nothing else belongs to $\mathscr{S}^{\dagger}$. The terms belonging to $\mathscr{S}^{\dagger}$ are canonical according to $\mathbf{P}^{K-\dagger}$.

Lemma 3.5. $\vdash_{\mathbf{P}^{K-\dagger}} \forall x \forall y(x=\mathbf{0} \rightarrow(\phi \rightarrow \phi(\mathbf{0} / x)))$.

Proof. The first step is the same as the first step of the proof of Lemma 3.1. The second step is essentially the same, since $\vdash_{\mathbf{P}} x+\mathbf{s}(\mathbf{0}) \neq \mathbf{0}$.

Lemma 3.6. $\vdash_{\mathbf{P}^{K-\dagger}} \forall x \forall y(x=y+\mathbf{s}(\mathbf{0}) \rightarrow(\phi \rightarrow \phi(y+\mathbf{s}(\mathbf{0}))))$.

Proof. The first step is the same as the first step of the proof of Lemma 3.2, since $\vdash_{\mathbf{P}} \mathbf{0} \neq y+\mathbf{s}(\mathbf{0})$. The second step is essentially the same as the second step of the proof of Lemma 3.2, since $\vdash_{\mathbf{P}} x+\mathbf{s}(\mathbf{0})=$ $y+\mathbf{s}(\mathbf{0}) \rightarrow x=y$.

Theorem 3.7. $\vdash_{\mathbf{P}^{K-\dagger}} \forall x \phi \rightarrow \phi(t / x)$ for all $t \in \mathscr{S}^{\dagger}$.

The next question is whether the Peano terms are still canonical according to $\mathbf{P}^{K-\dagger}$. As before, in case of a negative answer, one should check whether the answer remains the same if one uses a deductively stronger theory formulated in an extended language. For this end, consider the theory $\mathbf{M E P}^{\diamond K-\dagger}$, which is identical to $\mathbf{M E P}^{\diamond K-}$, except that IND is replaced by (18).

Theorem 3.8. $\nvdash_{\mathbf{M E P}} \diamond K-\dagger \forall x K x=x \rightarrow K \mathbf{s}(\mathbf{s}(\mathbf{0}))=\mathbf{s}(\mathbf{s}(\mathbf{0}))$.

Proof. Consider the model described in the proof of Theorem 3.4. Change the clause for $A\left(w_{2}, a\right)$ to: $\left\{t=t^{\prime} \mid \operatorname{den}_{\mathscr{N}, a}(t)=\operatorname{den}_{\mathscr{N}, a}\left(t^{\prime}\right)\right\}$ for every $t, t^{\prime} \in \mathscr{S}^{\dagger}$. It is easy to check that $\mathscr{M}, w_{2}=\forall x K x=x$, but $\mathscr{M}, w_{2} \not \models K \mathbf{s}(\mathbf{s}(\mathbf{0}))=\mathbf{s}(\mathbf{s}(\mathbf{0}))$. One should also verify that $\mathscr{M} \models(18)$. For this it is crucial that one can prove a version of the third replacement principle.

PR3 $^{\dagger}$ For all $\phi \in \mathscr{L}_{\mathbf{A}} \cup\{\diamond, K\}$, for all worlds $w$ and assignments $a$, for any variable $x$ and term $t \in S^{\dagger}$, it is the case that $\mathscr{M}, w, a \models \phi(t / x)$ if and only if $\mathscr{M}, w, a\left[x \mapsto \operatorname{den}_{\mathscr{M}, a}(t)\right] \models \phi$

The details are left to the reader.

A consequence of Theorems 3.7 and 3.8 is that the framework under consideration is highly nonextensional in that even provable identities are not always substitutable. For example,

$$
\nvdash_{\mathbf{P}^{K-\dagger}} \forall x K x=x \rightarrow K \mathbf{s}(\mathbf{s}(\mathbf{0}))=\mathbf{s}(\mathbf{s}(\mathbf{0}))
$$

but

$$
\vdash_{\mathbf{P}^{K-\dagger}} \forall x K x=x \rightarrow K \mathbf{0}+\mathbf{s}(\mathbf{0})+\mathbf{s}(\mathbf{0})=\mathbf{0}+\mathbf{s}(\mathbf{0})+\mathbf{s}(\mathbf{0}),
$$

despite the provability of $\mathbf{s}(\mathbf{s}(\mathbf{0}))=\mathbf{0}+\mathbf{s}(\mathbf{0})+\mathbf{s}(\mathbf{0})$.

The classical induction scheme IND suffices to prove that the Peano terms, e.g. $\mathbf{s}(\mathbf{s}(\mathbf{0}))$, are canonical, whereas it does not suffice to prove that the other arithmetical terms, e.g. $\mathbf{0}+\mathbf{s}(\mathbf{0})+\mathbf{s}(\mathbf{0})$, are not canonical. Likewise, the alternative, but extensionally equivalent induction scheme (18) suffices to prove that, e.g., $\mathbf{0}+\mathbf{s}(\mathbf{0})+\mathbf{s}(\mathbf{0})$, is canonical, whereas it does not suffice to prove that the Peano terms other than $\mathbf{0}$, e.g. $\mathbf{s}(\mathbf{s}(\mathbf{0}))$, are canonical. This variation seems to be explicable by the fact that the classical induction scheme IND contains $\mathbf{0}$ and $\mathbf{s}(x)$ but not $x+\mathbf{s}(\mathbf{0})$, whereas the induction scheme (18) contains $\mathbf{0}$ and $x+\mathbf{s}(\mathbf{0})$ but not $\mathbf{s}(x)$. I will now show that this line of explanation is too simple. 


\subsubsection{Alternative induction schemes and canonicity}

If one had UIt or SUBt, then (15) and (16) would be provably equivalent to:

$$
\begin{array}{r}
\forall x(x=\mathbf{0} \rightarrow \boldsymbol{\phi}) ; \\
\forall x(\phi \rightarrow \forall y(y=\mathbf{s}(x) \rightarrow \phi(y / x))) .
\end{array}
$$

In that scenario IND would be provably equivalent to

$$
\begin{array}{r}
(\phi(\mathbf{0} / x) \wedge \forall x(\phi \rightarrow \forall y(y=\mathbf{s}(x) \rightarrow \phi(y / x)))) \rightarrow \forall x \phi ; \\
(\forall x(x=\mathbf{0} \rightarrow \phi) \wedge \forall x(\phi \rightarrow \phi(\mathbf{s}(x) / x))) \rightarrow \forall x \phi ; \\
(\forall x(x=\mathbf{0} \rightarrow \phi) \wedge \forall x(\phi \rightarrow \forall y(y=\mathbf{s}(x)) \rightarrow \phi(y / x))) \rightarrow \forall x \phi .
\end{array}
$$

So, in extensional logic (in which SUBt is valid), IND is provably equivalent to (21) -(23). Note that, if $\phi$ is a purely arithmetical formula, then IND is provably equivalent to (21)-(23), assuming RUIt.

Let $\mathbf{P}^{K-*}$ be identical to $\mathbf{P}^{K-}$, except that the classical induction scheme IND is replaced by (21), i.e. with the scheme in which (16) has been replaced by (20). Let $\mathbf{P}^{K-* *}$ be identical to $\mathbf{P}^{K-}$, except that the classical induction scheme IND is replaced by (22), i.e. with the scheme in which (15) has been replaced by (19). Then one can prove the following counterparts of Lemmas 3.1 and 3.2.

Lemma 3.9. $\vdash_{\mathbf{p}^{K-*}} \forall x(x=\mathbf{0} \rightarrow(\phi \rightarrow \phi(\mathbf{0} / x)))$.

Proof. The first step is the same as the first step of the proof of Lemma 3.1. The second step is essentially the same: the antecedent of $\forall y(y=\mathbf{s}(x) \rightarrow(y=\mathbf{0} \rightarrow \ldots))$ contradicts the $\mathbf{P}$-axiom $\forall x \mathbf{s}(x) \neq \mathbf{0}$ just as well.

Lemma 3.10. $\vdash_{\mathbf{P}^{K-* *}} \forall x \forall y(x=\mathbf{s}(y) \rightarrow(\phi \rightarrow \phi(\mathbf{s}(y) / x)))$ (assuming that $\mathbf{s}(y)$ is substitutable for $x$ in $y)$.

Proof. The first step is essentially the same as the first step of the proof of Lemma 3.2: the antecedent of $\forall z(z=\mathbf{0} \rightarrow \forall y(z=\mathbf{s}(y) \rightarrow \ldots))$ contradicts $\forall y \mathbf{0} \neq \mathbf{s}(y)$ just as well. The second step is the same as the second step of the proof of Lemma 3.2.

So far, so good. The natural follow-up questions are whether $\mathbf{P}^{K-*}$ and $\mathbf{P}^{K-* *}$ prove the counterparts of Lemma 3.2 and Lemma 3.1 respectively. As before, in case of a negative answer, one should check whether the answer remains the same if one uses a deductively stronger theory formulated in an extended language. For this end variations on the theory considered in Subsection 3.2 will be used.

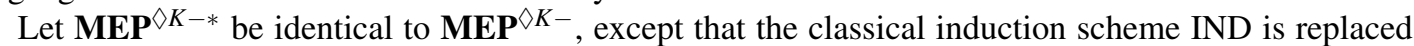
by (21), i.e. with the scheme in which (16) has been replaced by (20). Let $\mathbf{M E P} \mathbf{P}^{\diamond K-* *}$ be identical to $\mathbf{M E P}^{\diamond K-}$, except that the classical induction scheme IND is replaced by (22), i.e. with the scheme in

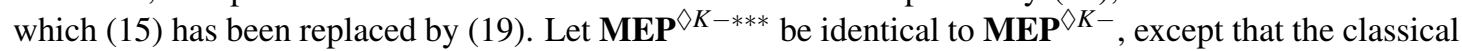
induction scheme IND is replaced by (23), i.e. with the scheme in which (15) and (16) have been replaced by (19) and (20) respectively.

Theorem 3.11. $\nvdash$ MEA $^{*} \forall x K x=x \rightarrow K \mathbf{s}(\mathbf{0})=\mathbf{s}(\mathbf{0})$.

Proof. Consider the model described in the proof of Theorem 3.4. Change the clause for $A\left(w_{2}, a\right)$ to: $\left\{t=t^{\prime} \mid \operatorname{den}_{\mathscr{N}, a}(t)=\operatorname{den}_{\mathscr{N}, a}\left(t^{\prime}\right)\right\}$ for every $t, t^{\prime}$ that are variables or $\mathbf{0}$. Call the latter set $\mathscr{S}^{*}$. It is easy to check that $\mathscr{M}, w_{2} \models \forall x K x=x$, but $\mathscr{M}, w_{2} \not \models K \mathbf{s}(\mathbf{0})=\mathbf{s}(\mathbf{0})$. One should also verify that $\mathscr{M} \models(21)$. For this it is crucial that one can prove another variation on the third replacement principle.

PR3 $^{*}$ For all $\phi \in \mathscr{L}_{\mathbf{A}} \cup\{\diamond, K\}$, for all worlds $w$ and assignments $a$, for any variable $x$ and term $t \in S^{*}$, it is the case that $\mathscr{M}, w, a \models \phi(t / x)$ if and only if $\mathscr{M}, w, a\left[x \mapsto \operatorname{den}_{\mathscr{M}, a}(t)\right] \models \phi$. 
The details are left to the reader.

Theorem 3.12. $\nvdash$ MEA $^{* *} \forall x K x=x \rightarrow K \mathbf{0}=\mathbf{0}$.

Proof. Consider the model described in the proof of Theorem 3.4. Change the clause for $A\left(w_{2}, a\right)$ to: $\left\{t=t^{\prime} \mid \operatorname{den}_{\mathscr{N}, a}(t)=\operatorname{den}_{\mathscr{N}, a}\left(t^{\prime}\right)\right\}$ for every $t, t^{\prime}$ that belong to the following inductively defined set $\mathscr{S}^{* *}$ : if $t$ is a variable, then it belongs to $\mathscr{S}^{* *}$; if $t^{\prime}$ belongs to $\mathscr{S}^{* *}$, then so does $\mathbf{s}\left(t^{\prime}\right)$; nothing else belongs to $\mathscr{S}^{* *}$. It is easy to check that $\mathscr{M}, w_{2} \models \forall x K x=x$, but $\mathscr{M}, w_{2} \not \models K \mathbf{0}=\mathbf{0}$. One should also verify that $\mathscr{M} \models(22)$. For this it is crucial that one can prove yet another variation on the third replacement principle.

PR3 $^{* *}$ For all $\phi \in \mathscr{L}_{\mathbf{A}} \cup\{\diamond, K\}$, for all worlds $w$ and assignments $a$, for any variable $x$ and term $t \in \mathscr{S}^{* *}$, it is the case that $\mathscr{M}, w, a \mid=\phi(t / x)$ if and only if $\mathscr{M}, w, a\left[x \mapsto \operatorname{den}_{\mathscr{M}, a}(t)\right] \models \phi$.

The details are left to the reader.

Theorem 3.13. $\nvdash$ MEA $^{* * *} \forall x K x=x \rightarrow(K \mathbf{0}=\mathbf{0} \wedge K \mathbf{s}(\mathbf{0})=\mathbf{s}(\mathbf{0}))$.

Proof. Consider the model described in the proof of Theorem 3.4. Change the clause for $A\left(w_{2}, a\right)$ to: $\{x=y \mid a(x)=a(y)\}$ for any variables $x, y$. It is easy to check that $\mathscr{M}, w_{2} \models \forall x K x=x$, but $\mathscr{M}, w_{2} \not \models$ $K \mathbf{0}=\mathbf{0}$ and $\mathscr{M}, w_{2} \not \models K \mathbf{s}(\mathbf{0})=\mathbf{s}(\mathbf{0})$. One should also verify that $\mathscr{M} \models(23)$. For this it is crucial that one can prove one more variation on the third replacement principle.

PR3 $^{* * *}$ For all $\phi \in \mathscr{L}_{\mathbf{A}} \cup\{\diamond, K\}$, for all worlds $w$ and assignments $a$, for any variables $x, y$, it is the case that $\mathscr{M}, w, a \models \phi(y / x)$ if and only if $\mathscr{M}, w, a[x \mapsto a(y)] \models \phi$.

The details are left to the reader.

A consequence of Theorems 3.11-3.13 is that the frameworks in question are non-extensional in that material identities are not always substitutable, e.g.

$$
\forall \mathbf{p}^{K-*} x=\mathbf{s}(\mathbf{0}) \rightarrow(K x=x \rightarrow K \mathbf{s}(\mathbf{0})=\mathbf{s}(\mathbf{0}))
$$

and

$$
\nvdash_{\mathbf{P}^{K-* *}} x=\mathbf{0} \rightarrow(K x=x \rightarrow K \mathbf{0}=\mathbf{0}) .
$$

The take-home lesson of the above theorems is that it is not enough that the symbol for zero and the symbol for the successor appear in the induction scheme. Extensionally equivalent formulations of the induction scheme, viz. (21)-(23), which still contain $\mathbf{0}$ and $\mathbf{s}$, do not suffice to prove that Peano terms are canonical.

\subsection{A potential explanation for the canonicity of Peano numerals}

In order to not lose sight of the wood for the trees I will recapitulate the main findings so far. It was shown that allowing the knowledge operator to occur in instances of the induction scheme yields the eligibility of Peano terms for universal instantiation and existential generalisation in epistemic contexts. Then it was demonstrated that one does not get the result that other arithmetical terms are similarly privileged, even if one adds certain modal, epistemic and modal-epistemic principles. The source of these two (non-)canonicity results was tentatively located in the fact that the induction scheme contains the Peano numeral for zero and the symbol for the successor function. Further evidence for this was found in the fact that, if one were to replace $\mathbf{s}(x)$ in the induction scheme with $x+\mathbf{s}(\mathbf{0})$, then Peano numerals of the form $\mathbf{s}(t)$ with $t$ a Peano numeral are no longer canonical terms, whereas terms of $\mathscr{T}($ e.g., $\mathbf{0}+\mathbf{s}(\mathbf{0})$ ) become canonical. It was noted that the framework used is highly non-extensional, since even provably 
co-denoting terms are not substitutable in epistemic contexts. Then I presented counterevidence: there are alternative induction schemes that also contain the symbols for zero and the successor relation but that do not entail the canonicity of Peano numerals. It was noted that the frameworks used are non-extensional in that co-denoting terms are not substitutable in epistemic contexts. The upshot of this investigation is that it is the induction scheme in its standard form that is at the basis of the canonicity of Peano numerals.

On the one hand, we have found an explanation for the canonicity of Peano numerals. According to a simple version of the deductive model of explanation (Hempel and Oppenheim, 1948), ${ }^{4}$ an explanans explains an explanandum relative to a background theory if and only if, first, the explanans logically entails the explanandum relative to the background theory, second, the background theory alone does not logically entail the explanandum and, third, the explanans is true. If the first two requirements are satisfied, then there is a potential explanation.

The schema $\operatorname{IND}^{K}$ (i.e., the induction scheme with formulas belonging to $\mathscr{L}_{\mathbf{A}} \cup\{K\}$ ) is the explanans, the schema

$$
\forall x \phi \rightarrow \phi(t / x) \quad \text { for all Peano terms } t
$$

is the explanandum and, say, $\mathbf{R}^{K-}$ (i.e., $\mathbf{R}$ formulated in $\mathscr{L}_{\mathbf{A}} \cup\{K\}$ and with UI and RUIt rather than UIt and with SUB rather than SUBt) is the background theory. Note that the extension of $\mathbf{R}^{K-}$ with $\mathrm{IND}^{K}$ yields $\mathbf{P}^{K-}$. Next it needs to be verified that the three requirements on deductive explanations are satisfied. $^{5}$

It was shown in Subsection 3.1 that $\mathbf{P}^{K-} \vdash$ (24) (cf. Theorem 3.3), so the first requirement is satisfied. For the second requirement it needs to be shown that $\mathbf{R}^{K-} \nvdash(24)$.

Theorem 3.14. $\mathbf{R}^{K-} \nvdash(24)$.

Proof. Consider again the model described in the proof of Theorem 3.4. Drop $W, R_{M}, R_{E}$. Define $A(a)$ as $\{x=y \mid a(x)=a(y)\}$ (compare to the proof of Theorem 3.13). Satisfaction is no longer relative to elements of $W$. The satisfaction clause for $\phi=K \psi$ changes to: $\mathscr{M}, a \mid \phi$ if and only if $\mathscr{M}, a \models \psi$ and $\psi \in A(a)$. I leave it to the reader to verify that $\mathscr{M} \models \mathbf{R}^{K-}$. Finally, I it is easy to check that $\mathscr{M} \models \forall x K x=x$, while $\mathscr{M} \forall K \mathbf{0}=\mathbf{0}$.

At this point it has already been established that $\operatorname{IND}^{K}$ is a potential explanation of (24). The third requirement is that $\mathrm{IND}^{K}$ is true. If instantiations of the induction scheme with formulas that contain the knowledge operator do not result in falsehoods, then the requirement is satisfied as well. I do not have a knock-down argument here, but I would like to point to a couple of facts. First, $\mathbf{P}^{K-}$ is a consistent extension of $\mathbf{P}$. Second, $\mathbf{P}^{K-}$ is an arithmetically conservative extension of $\mathbf{P}$ and, hence, $\mathbf{P}^{K-}$ is arithmetically sound. (Both facts can be proved with the help of the eraser translation.) This is not enough to conclude that IND ${ }^{K}$ is philosophically (epistemically) sound as well. Yet it confers some legitimacy. I will briefly return to this issue. To conclude, the full induction scheme serves at the very least as a potential deductive explanation of the canonicity of Peano numerals.

Building on the findings of Subsection 3.3, one can equally well argue that, if the terms belonging to $\mathscr{S}^{\dagger}$ were canonical, then alternative induction scheme (18) would constitute a good explanation for it. Similar remarks can be made about (21)-(23) and $\mathscr{S}^{*}, \mathscr{S}^{* *}, \mathscr{V}$ (with $\mathscr{V}$ the set of variables) respectively. Generally speaking, it was seen that the canonicity of certain sets of arithmetical terms covaries with variations on the induction scheme. This can be taken to be an indication of the dependence of canonicity on induction.

\footnotetext{
${ }^{4}$ The simplification consists in the fact that here it is not required that the explanans is a general empirical law. In the case at hand the explanans is a mathematical axiom scheme with an individual constant. Note also that I speak of an explanation relative to a background theory rather than include the background theory in the explanans.

${ }^{5}$ Technically speaking, in what follows I will assume that the explanans and/or the explanandum are not added as (sets of) hypotheses but as axioms (or rather, axiom schemes) to the background theory.
} 
On the other hand, there is an important limitation to the explanation offered in this section. The explanation given earlier is internal to the deductive framework that was used. As has been argued in Subsection 3.3, the exact form of the induction scheme matters. This raises the question why exactly $\mathrm{IND}^{K}$ is adopted. The latter question is external to the framework that was used. If one wants more than a potential deductive explanation, one needs a reason to accept that $\mathrm{IND}^{K}$ is true or, eschewing truth talk, one needs an external reason for using $\mathbf{P}^{K-}$ rather than, say, $\mathbf{P}^{K-\dagger}$.

Let us now return to two questions that were raised in Section 1. The first question pertained to the choice of the system of Peano numerals over other systems. To explain the canonicity of Peano numerals good use has been made of the axiomatic framework of Peano Arithmetic and, in particular, of the induction scheme with its Peano numeral for zero and the symbol for the successor function. An explanation of why the Hindu-Arabic numerals are buck stoppers can only go along the same lines if one, first, formulates a formal, axiomatic theory and, second, formulates an induction scheme with the help of the Hindu-Arabic numeral system. I have not worked out the details, but my guess is that this can be done by replacing the primitive symbol $\mathbf{s}$ with a defined symbol $\mathrm{s}$, with $\mathrm{s}(0)=1$ and so on for the other base numerals and subsequently for the sequences of Hindu-Arabic numerals. Then one could go on and attempt to prove a canonicity result. This is left for future work.

The second question was about how to solve the problem of big numerals. The result obtained in Subsection 3.1 says that the standard induction scheme entails the canonicity of Peano numerals (given certain background assumptions). If one does not accept large Peano numerals as canonical terms, then one has no choice but to reject the standard induction scheme formulated in $\mathscr{L}_{\mathbf{A}} \cup\{K\}$. This is an important constraint on possible solutions to the problem of large numerals. Moreover, if one goes down this path, the risk is that one loses the explanation for why small numerals are canonical.

\section{Arithmetical terms and indirect answers}

So far the focus was on Peano numerals and, more broadly, Peano terms being eligible for universal instantiation and existential generalisation in epistemic contexts. In this section the focus shifts to all arithmetical terms being eligible for universal instantiation and existential generalisation in contexts in which every occurrence of the knowledge operator is preceded by a possibility operator and every possibility operator is followed by a knowledge operator. In Subsection 4.1 it will be demonstrated that all closed arithmetical terms fit the bill and in Subsection 4.2 it will be argued that open arithmetical terms have this property as well. As before, the induction scheme plays an important role in obtaining these results. Subsection 4.3 focuses on an important consequence: order statements are knowable.

\subsection{Closed arithmetical terms}

It is provable that any closed arithmetical term is eligible for universal instantiation and existential generalization in contexts belonging to $\tau$.

Let $\mathbf{M E Q} \mathbf{Q}^{-}$be identical to $\mathbf{M E P} \mathbf{P}^{\diamond K-}$, except that $\mathbf{P}$ is replaced with minimal arithmetic $\mathbf{Q}$.

Lemma 4.1. If $\vdash_{\mathbf{Q}} t=t^{\prime}$, then $\vdash_{\mathbf{M E Q}^{-}} \phi \leftrightarrow \phi\left(t^{\prime} / t\right)$ for every $\phi \in \tau$ and for all $t \in \mathscr{L}_{\mathbf{A}}$ (assuming that $t^{\prime}$ is substitutable for $t$ in $\phi$ ).

Proof. The proof is by induction on $\phi$. The atomic case follows from SUB, UG, RUIt and the symmetry of identity. The case of $\phi=\neg \psi$ follows by contraposition from the induction hypothesis. The case of $\phi=\psi \wedge \theta$ is trivial as well. The case of $\phi=\forall x \psi$ follows by the induction hypothesis, UG and the definition of the substitutability of a term for a variable in a formula. The case of $\phi=\diamond K \psi$ follows from the induction hypothesis, COMP and S4.

Theorem 4.2. $\vdash_{\text {MEP }}^{\diamond K-} \forall x \phi \rightarrow \phi(t / x)$ for all $\phi \in \tau$ and for all closed $t \in \mathscr{L}_{\mathbf{A}}$. 
Proof. For every closed $t \in \mathscr{L}_{\mathbf{A}}$, there is a $n \in \mathbb{N}$ such that $\vdash_{\mathbf{Q}} t=\bar{n}$ and, hence, $\vdash_{\text {MEP }} \diamond-t=\bar{n}$. Theorem 3.3 implies that $\vdash_{\mathbf{M E P}}^{\diamond K_{-}} \forall x \phi \rightarrow \phi(\bar{n} / x)$ for any $n \in \mathbb{N}$. Then use Lemma 4.1 and the fact that MEQ $\mathbf{M}^{-} \subseteq$ $\mathbf{M E P}^{\diamond K-}$ to derive the desired conclusion.

Theorem 4.2 is restricted to closed terms, e.g. $\mathbf{s}(\mathbf{0}) \times \mathbf{0}$, while one would also like to know whether open terms, e.g. $x \times \mathbf{0}$, are eligible for universal instantiation and existential generalisation in $\tau$-contexts. The proof of Theorem 4.2 relied indirectly on the induction scheme by the fact that it invoked Theorem 3.3. The proofs used in the next subsection will make more direct use of the induction scheme.

\subsection{Any arithmetical terms}

One can prove that not only closed but also open arithmetical terms are eligible for universal instantiation and existential generalisation in $\tau$-contexts.

Corollary 4.3 (Lemma 3.2). $\vdash_{\mathbf{M E P}^{\diamond K-}} \forall x \phi \rightarrow \forall x \phi(\mathbf{s}(x))$.

Proof. Left to the reader.

Lemma 4.4. $\vdash_{\mathbf{M E P}^{\diamond K-}} \forall x \forall y \forall z(x=y+z \rightarrow(\phi \leftrightarrow \phi((y+z) / x)))$ for all $\phi \in \tau$.

Proof. The proof is by the induction principle.

First, one needs to prove that

$$
\forall x \forall z(x=\mathbf{0}+z \rightarrow(\phi \leftrightarrow \phi((\mathbf{0}+z) / x))) .
$$

It is an instantiation of SUB that $x=z \rightarrow(\phi \leftrightarrow \phi(z / x))$. It is a theorem of $\mathbf{P}$ that $z=\mathbf{0}+z$. Therefore, by Lemma 4.1, it follows that

$$
x=\mathbf{0}+z \rightarrow(\phi \leftrightarrow \phi((\mathbf{0}+z) / x)) .
$$

Second, one needs to prove that for all $y$, if

$$
\forall x \forall z(x=y+z \rightarrow(\phi \leftrightarrow \phi((y+z) / x)))
$$

then

$$
\forall x \forall z(x=\mathbf{s}(y)+z \rightarrow(\phi \leftrightarrow \phi((\mathbf{s}(y)+z) / x)))
$$

It is provable in $\mathbf{P}$ that

$$
\begin{aligned}
& \mathbf{s}(y)+z=\mathbf{s}(y+z) \\
& \mathbf{s}(y+z)=y+\mathbf{s}(z) \\
& \mathbf{s}(y)+z=y+\mathbf{s}(z)
\end{aligned}
$$

Hence, it follows from Corollary 4.3 and the antecedent of the induction hypothesis that

$$
\forall x \forall z(x=y+\mathbf{s}(z) \rightarrow(\phi \leftrightarrow \phi((y+\mathbf{s}(z)) / x))) .
$$

Given (27) and Lemma 4.1, it follows that

$$
x=\mathbf{s}(y)+z \rightarrow(\phi \leftrightarrow \phi((\mathbf{s}(y)+z) / x)) .
$$

Application of UG and conditionalisation are the remaining steps.

Corollary 4.5. $\vdash_{\mathbf{M E P}^{\diamond K-}} \forall x \phi \rightarrow \forall y \forall z \phi(y+z)$ for all $\phi \in \tau$. 
Proof. Left to the reader.

Lemma 4.6. $\vdash_{\mathbf{M E P}^{\diamond K-}} \forall x \forall y \forall z(x=(y \times z)+z \rightarrow(\phi \leftrightarrow \phi(((y \times z)+z) / x)))$ for all $\phi \in \tau$.

Proof. The proof is by the induction principle.

First, one needs to prove that

$$
\forall x \forall z(x=(\mathbf{0} \times z)+z \rightarrow(\phi \leftrightarrow \phi(((\mathbf{0} \times z)+z) / x))) .
$$

Suppose that $x=(\mathbf{0} \times z)+z$. It is a theorem of $\mathbf{P}$ that $\mathbf{0} \times z=\mathbf{0}$. Therefore, one can use Lemma 4.1 to deduce $x=\mathbf{0}+z$. It is a consequence of the foregoing and Lemma 4.4 and Theorem 3.3 that $\phi \leftrightarrow$ $\phi((\mathbf{0}+z) / x)$. Given the fact that $\vdash_{\mathbf{P}} \mathbf{0} \times z=\mathbf{0}$ and Lemma 4.1, it follows that $\phi \leftrightarrow \phi(((\mathbf{0} \times z)+z) / x)$. Second, one has to prove that, if

$$
\forall x \forall z(x=(y \times z)+z \rightarrow(\phi \leftrightarrow \phi(((y \times z)+z) / x))),
$$

then

$$
\forall x \forall z(x=(\mathbf{s}(y) \times z)+z \rightarrow(\phi \leftrightarrow \phi(((\mathbf{s}(y) \times z)+z) / x))) .
$$

It is a theorem of $\mathbf{P}$ that $\mathbf{s}(y) \times z=(y \times z)+z$ and, consequently, $(\mathbf{s}(y) \times z)+z=(y \times z)+z+z$. Moreover, is a consequence of Corollary 4.5 that $\forall x \phi \rightarrow \forall y \phi(y+y / x)$. Hence, it follows from the induction hypothesis that

$$
\forall x \forall z(x=(y \times z)+z+z \rightarrow(\phi \leftrightarrow \phi(((y \times z)+z+z) / x))) .
$$

Since $\vdash_{\mathbf{P}}(\mathbf{s}(y) \times z)+z=(y \times z)+z+z$, one can use Lemma 4.1 to derive that

$$
\forall x \forall z(x=(\mathbf{s}(y) \times z)+z \rightarrow(\phi \leftrightarrow \phi(((\mathbf{s}(y) \times z)+z) / x))) .
$$

Conditionalisation and UG do the rest.

Lemma 4.7. $\vdash_{\mathbf{M E P}^{\diamond K-}} \forall x \forall y \forall z(x=y \times z \rightarrow(\phi \leftrightarrow \phi((y \times z) / x)))$ for all $\phi \in \tau$.

Proof. The proof is by the induction principle.

First, one needs to prove that

$$
\forall x \forall z(x=\mathbf{0} \times z \rightarrow(\phi \leftrightarrow \phi((\mathbf{0} \times z) / x))) .
$$

Suppose that $x=\mathbf{0} \times z$. It is a theorem of $\mathbf{P}$ that $\mathbf{0}=\mathbf{0} \times z$. It follows that $x=\mathbf{0}$. By Lemma 3.1, it is derivable that $\phi \leftrightarrow \phi(\mathbf{0} / x)$. Lemma 4.1 and the aforementioned theorem of $\mathbf{P}$ then entail that $\phi \leftrightarrow$ $\phi((\mathbf{0} \times z) / x)$.

Second, one has to prove that for all $y$, if

$$
\forall x \forall z(x=y \times z \rightarrow(\phi \leftrightarrow \phi((y \times z) / x))),
$$

then

$$
\forall x \forall z(x=\mathbf{s}(y) \times z \rightarrow(\phi \leftrightarrow \phi((\mathbf{s}(y) \times z) / x))) .
$$

Suppose that $x=\mathbf{s}(y) \times z$. It is a theorem of $\mathbf{P}$ that $\mathbf{s}(y) \times z=(y \times z)+z$. It is an instantiation of of Lemma 4.6 that $x=(y \times z)+z \rightarrow(\phi \rightarrow \phi(((y \times z)+z) / x))$. By Lemma 4.1, it follows that $x=(\mathbf{s}(y) \times z) \rightarrow$ $(\phi \rightarrow \phi((\mathbf{s}(y) \times z) / x))$.

Theorem 4.8. $\vdash_{\mathbf{M E P}^{\diamond K-}} \forall x \phi \rightarrow \phi(t / x)$ for all $\phi \in \tau$ and for all $t \in \mathscr{L}_{\mathbf{A}}$. 
Proof. Left to the reader.

This answers the question at the end of Subsection 4.1, namely $x \times \mathbf{0}$ is also eligible for universal instantiation and existential generalisation in $\tau$-contexts, just as $\mathbf{s}(\mathbf{0}) \times \mathbf{0}$ is.

At this point the question arises whether IND ${ }^{\diamond K}$ provides a deductive explanation of

$$
\forall x \phi \rightarrow \phi(t / x) \quad \text { for all } \phi \in \tau \text { and for all } t \in \mathscr{L}_{\mathbf{A}} .
$$

The explanandum is (28), the explanans is $\mathrm{IND}^{\diamond K}$ and, say, $\mathbf{M E \mathbf { Q } ^ { - }}$ is the background theory. The first requirement on deductive explanations is satisfied: Theorem 4.8 says that $\mathbf{M E P}^{\diamond K-} \vdash$ (28). It is an open question whether the second requirement is satisfied. In other words, I don't have a proof of MEQ $^{-} \nvdash(28)$. So, it has not been established that $\mathrm{IND}^{K}$ is a (potential) explanans of the eligibility of all arithmetical terms for universal instantiation and existential generalisation in certain modal-epistemic contexts, although one important element has been provided.

Theorem 4.8 has a couple of interesting consequences. These are the topic of the next subsection.

\subsection{The knowability of order statements}

In Section 2 it was pointed out that the decidability of order statements with Peano numerals and other terms has philosophical significance. Clearly, one can express this in the language $\mathscr{L}_{\mathbf{A}} \cup\{\oslash, K\}$ as follows:

$$
\begin{aligned}
& t=t^{\prime} \rightarrow \diamond K t=t^{\prime} ; \\
& t<t^{\prime} \rightarrow \diamond K t<t^{\prime} .
\end{aligned}
$$

The further question is whether one can also prove it in $\mathbf{M E P}^{\diamond K-}$. Before backing up the affirmative answer to this last question, I will briefly explain how this approach differs from a more widely known approach.

As has been claimed several times before, for any closed terms $t$ and $t^{\prime}$, if $\mathscr{N} \models t=t^{\prime}$, then $\vdash_{\mathbf{Q}} t=t^{\prime}$. There is a rudimentary formula $\operatorname{Pr} f_{\mathbf{Q}}\left(\left\ulcorner t=t^{\prime}\right\urcorner, y\right)$ (with $\left\ulcorner t=t^{\prime}\right\urcorner$ the Gödel code of the sentence $t=t^{\prime}$ ) such that it follows from the latter that $\vdash_{\mathbf{Q}} \exists y \operatorname{Prf}_{\mathbf{Q}}\left(\left\ulcorner t=t^{\prime}\right\urcorner, y\right)$. Whence it follows that $\vdash_{\mathbf{Q}} t=t^{\prime} \rightarrow$ $\exists y \operatorname{Prf}_{\mathbf{Q}}\left(\left\ulcorner t=t^{\prime}\right\urcorner, y\right)$. Likewise for $t<t^{\prime}$. The details can be found in (Boolos et al, 2003). Ergo, one can already express in $\mathscr{L}_{\mathbf{A}}$ and prove in $\mathbf{Q}$ the provability of order statements with closed terms. Yet, this proceeds via Gödel coding, whereas no such coding is needed in what follows. Moreover, the proof predicate for $\mathbf{Q}$ is not factive (Boolos et al, 2003, p. 234-238), whereas the knowledge operator is. So the exercise undertaken in this subsection is not pointless.

The affirmative answer alluded to earlier is to a large extent a consequence of Theorem 4.8.

Corollary 4.9 (Theorem 4.8). $\vdash_{\text {MEP }^{\diamond K-}} t=t^{\prime} \rightarrow \diamond K t=t^{\prime}$.

Proof. An instantiation of SUB is the following:

$$
x=y \rightarrow(\diamond K x=x \rightarrow \diamond K x=y) .
$$

Use COMP to derive from SI that $\diamond K x=x$. Hence,

$$
x=y \rightarrow \diamond K x=y .
$$

Then use UG and Theorem 4.8 to derive the desired conclusion.

Theorem 4.10. $\vdash_{\mathbf{M E P}^{\diamond K-}} \forall x \forall y(x<y \rightarrow \diamond K x<y)$. 
Proof. The proof is by the induction principle. ${ }^{6}$

First, one needs to prove that

$$
\forall x(x<\mathbf{0} \rightarrow \diamond K x<\mathbf{0}) .
$$

This follows from the axiom that $\neg \exists x(x<\mathbf{0})$.

Second, one needs to prove that

$$
\forall y(\forall x(x<y \rightarrow \diamond K x<y) \rightarrow \forall x(x<\mathbf{s}(y) \rightarrow \diamond K x<\mathbf{s}(y))) .
$$

It is an axiom that $x<\mathbf{s}(y) \leftrightarrow(x=y \vee x<y)$. It is a theorem that $y<\mathbf{s}(y)$ and, hence by COMP, $\diamond K y<\mathbf{s}(y)$. It follows by SUB that $\diamond K x<\mathbf{s}(y)$. Suppose that $x<y$. Then use the induction hypothesis and UG to infer that $\diamond K x<y$. It is a theorem that $x<y \rightarrow x<\mathbf{s}(y)$. It follows by COMP and S4 that $\diamond K x<y \rightarrow \diamond K x<\mathbf{s}(y)$. An application of modus ponens does the rest.

Corollary 4.11. $\vdash_{\mathrm{MEP}^{\vee} K-} t<t^{\prime} \rightarrow \diamond K t<t^{\prime}$.

Proof. From Theorems 4.10 and 4.8 .

On the one hand, the knowability of order statements is provable in $\mathbf{M E P} \mathbf{P}^{\diamond K-} .{ }^{7}$ On the other hand, it is not the case that order relations even between Peano numerals are known.

Theorem 4.12. $\nvdash_{\mathrm{MEP}^{\curlyvee K-}} \mathbf{0}=\mathbf{0} \rightarrow K \mathbf{0}=\mathbf{0}$.

Proof. Consider the model described in the proof of Theorem 3.4. Change the clause for $A\left(w_{2}, a\right)$ to $\emptyset$.

Theorem 4.13. $\nvdash_{\mathrm{MEP}} \backslash K-\mathbf{0}<\mathbf{s}(\mathbf{0}) \rightarrow K \mathbf{0}<\mathbf{s}(\mathbf{0})$.

Proof. Consider the model described in the proof of Theorem 3.4. Change the clause for $A\left(w_{2}, a\right)$ to $\emptyset$.

So, notwithstanding the fact that Peano numerals are canonical, order statements with only Peano numerals are not always known, although they are knowable.

\section{Conclusion}

Two goals were set out in Sections 1 and 2. The first goal was to shed some light on why Peano numerals are canonical terms, i.e. terms that are eligible for existential generalisation in epistemic contexts. In other words, the intimate connection between Peano numerals and de re knowledge about natural numbers was in need of an explanation. The second goal was to illuminate why all arithemetical terms can be used for indirect answers to questions about numbers or are eligible for existential quantification in certain modal-epistemic contexts, called $\tau$-contexts. In other words, the connection between arithmetical terms and possible de re knowledge about natural numbers was open for investigation.

It was argued that the full standard induction scheme provides a potential deductive explanation for the canonicity of Peano numerals. With the standard induction scheme, one can deduce that Peano numerals

\footnotetext{
${ }^{6}$ The proof is almost identical to the proof in (Heylen, 2013, p. 95), with the box operator replaced by the knowledge operator.

${ }^{7}$ In (Horsten, 2005, p. 249-251) and (Heylen, 2009, p. 224-225) it is proved that in Carnap-style intensional arithmetic identity statements and distinctness statements are knowable if true. In (Horsten, 2005) the second-order axiom of induction is used, whereas in (Heylen, 2009) the first-order induction scheme is used. See (Heylen, 2010, p. 370, fn. 9) for a more detailed comparison between the results. A major difference between the proofs in (Horsten, 2005) and (Heylen, 2009) on the one hand and the proof here is that the former make use of the postulate that Peano terms can be used in existential generalisation, whereas that is a theorem in the framework used here. Also interesting from the perspective of this paper are the discussions in (Horsten, 2005, p. 256 fn. 11) and (Heylen, 2009, p. 222-229, 231-232) of the soundness of induction in intensional arithmetic.
} 
are canonical (Theorem 3.3). Without it, one cannot (Theorem 3.14). So, the full standard induction scheme provides at least a potential explantion for the canonicity of Peano numerals. Moreover, variations on the induction scheme lead to variations in which sets of terms are canonical. However, the question of the truth of the full standard induction scheme and the external question of why to prefer it over other schemes has not been answered (Subsection 3.4).

It was also argued that the full standard induction scheme provides a first step in a deductive explanation for the eligibility of all arithmetical terms in $\tau$-contexts. With it, one can deduce the eligibility result (Theorem 4.8). An important open question is whether the full standard induction scheme is an essential ingredient in explaining why all arithmetical terms are eligible for universal instantiation and existential generalisation in $\tau$-contexts. An affirmative answer is needed for thinking that the full standard induction scheme provides a potential explanation.

This article focused on Peano numerals rather than Hindu-Arabic numerals as canonical names for numbers. The reasons for this are explained in Section 2 and Subsection 3.4. It is an open question whether the same explanation strategy can be used to explain the canonicity of Hindu-Arabic numerals. Another limitation of this article is that the problem of big numerals has not been solved, although the result obtained in Subsection 3.1 is claimed to be an important restriction on possible solutions to that problem (Subsection 3.4).

An important subtheme is non-extensionality (Subsection 3.3). Even provable identities are not always substitutable in epistemic contexts. Finally, it was shown that order statements are knowable, although even order statements with only Peano numerals are not always known (Subsection 4.3).

\section{Acknowledgements}

A previous version has been presented on the Workshop on Intensionality in Mathematics, which took place on 11-12 May, 2013 in Kungshuset, Lundagard, Sweden. I would like to thank Marianna AntonuttiMarfori, Carlo Proietti and Paula Quinon for organizing the workshop and for providing me with the opportunity to present my research and I am grateful to the audience for their feedback. Another version has been presented on May, 16, 2014 in Leuven, Belgium. Again I would like to thank the audience for their feedback. In addition, I would like to thank my colleagues Lorenz Demey, Markus Eronen and Harmen Ghijsen for reading and commenting on my paper. Also, I would like to thank two anonymous reviewers for their comments and suggestions. Finally, I would like to thank Marianna Antonutti-Marfori and Paula Quinon for editing this volume and for their careful reading of and useful feedback on various versions of this article.

\section{References}

Ackerman D (1978) De re propositional attitudes toward integers. Southwestern Journal of Philosophy $9(2): 145-153$

Boolos GS, Burgess JP, Jeffrey RC (2003) Computability and Logic, 4th edn. Cambridge University Press, New York

Button T, Smith P (2011) The philosophical significance of tennenbaum's theorem. Philosophia Mathematica

Carnap R (1956) Meaning and Necessity, 2nd edn. The University of Chicago Press, Chicago

Dean W (2013) Models and computability. Philosophia Mathematica DOI 10.1093/philmat/nkt035, URL http://philmat.oxfordjournals.org/content/early/2013/12/23/philmat.nkt035.abstract, http://philmat.oxfordjournals.org/content/early/2013/12/23/philmat.nkt035.full.pdf+html 
Halbach V, Horsten L (2005) Computational structuralism. Philosophia Mathematica 13(2):174-186

Hempel CG, Oppenheim P (1948) Studies in the logic of explanation. Philosophy of Science 15(2):135175

Heylen J (2009) Carnapian modal and epistemic arithmetic. In: Carrara M, Morato V (eds) Language, Knowledge, and Metaphysics. Proceedings of the First SIFA Graduate Conference, College Publications, London, pp 97-121

Heylen J (2010) Carnap's theory of descriptions and its problems. Studia Logica 94(3):355-380

Heylen J (2013) Modal-epistemic arithmetic and the problem of quantifying in. Synthese 190(1):89-111

Heylen J (2014) Closure of a priori knowability under a priori knowable material implication. Erkenntnis pp 1-22, DOI 10.1007/s10670-014-9647-4, URL http://dx.doi.org/10.1007/s10670-014-9647-4

Horsten L (1994) Modal-Epistemic variants of Shapiro's system of Epistemic Arithmetic. Notre Dame Journal of Formal Logic 35(2):284-291

Horsten L (1998) In defense of Epistemic Arithmetic. Synthese 116(1):1-25

Horsten L (2005) Canonical naming systems. Minds and Machines 15(2):229-257

Horsten L (2012) Vom Zählen zu den Zahlen: On the relation between computation and arithmetical structuralism. Philosophia Mathematica 20(3):275-288

Kaplan D (1968) Quantifying in. Synthese 19(1-2):178-214

Kripke SA (2011) Philosophical Troubles. Collected Papers Vol I. Oxford University Press

Quine WV (1956) Quantifiers and propositional attitudes. Journal of Philosophy 53(5):177-187

Quinon P (2014) Church's Thesis, Logic, Mind and Nature, Copernicus Center Press, chap From Computability over Strings of Characters to Natural Numbers

Quinon P, Zdanowski K (2007) The intended model of arithmetic. an argument from Tennenbaum's theorem. In: Cooper SB, Löwe B, Sorbi A (eds) Computation and Logic in the Real World, Springer, Lecture Notes in Computer Science, vol 4497

Russell B (1905) On denoting. Mind 14(56):479-493

Steiner M (2011) Saul Kripke, Cambridge University Press, chap Kripke on Logicism, Wittgenstein, and De Re Beliefs about Numbers, pp 160-176

Wittgenstein L (1978) Remarks on the Foundations of Mathematics, vol 7. B. Blackwell 